\title{
¿CÓMO FUNCIONA LA TERAPIA NARRATIVA? UNA REVISIÓN DE LA INVESTIGACIÓN DE PROCESOS Y PROCESOS-RESULTADOS
}

\section{HOW DOES NARRATIVE THERAPY WORK? \\ A REVIEW OF PROCESSES AND PROCESS-OUTCOME RESEARCH}

\author{
Danilo Moggia \\ Doctor en Psicología \\ Psicólogo General Sanitario \\ Psicoterapeuta \\ ORCID: https://orcid.org/0000-0001-6321-4450
}

Cómo referenciar este artículo/How to reference this article:

Moggia, D. (2019). ¿Cómo funciona la terapia narrativa? Una revisión de la investigación de procesos y procesos-resultados. Revista de Psicoterapia, 30(114), 31-55. https://doi.org/10.33898/rdp.v30i114.319

\section{Resumen}

El presente artículo es una revisión narrativa que sintetiza las principales investigaciones publicadas sobre procesos y procesos-resultados sobre terapia narrativa (TN). En primer lugar, se revisa brevemente la investigación de resultados que hay sobre la TN buscando responder a la pregunta si la TN funciona. En segundo lugar, se revisan las principales investigaciones de procesos y procesos-resultados profundizando en dos programas de investigación: los Momentos Innovadores y la Colaboración Terapéutica. Por último, se extraen conclusiones acerca de cómo funciona la TN a partir de las investigaciones expuestas.

Palabras clave: terapia narrativa, investigación en psicoterapia, investigación de procesos, investigación de procesos-resultados.

\begin{abstract}
The current article is a narrative review which summarises the main published studies on processes and process-outcome researchabout narrative therapy(NT). First, outcome research on NT is briefly reviewed with the aim of answering the question does NT works. Second, we reviewed the main published work on processes and process-outcomes research about NT, deepening in two research programmes: The Innovative Moments and The Therapeutic Collaboration. Finally, conclusions regarding how NT works are drawn.

Keywords: narrative therapy, psychotherapy research, processes research, process-outcome research.
\end{abstract}


Actualmente la investigación en psicoterapia puede ser dividida en cinco líneas principales (Gelo, Pritz y Rieken, 2015): la investigación de resultados, la de procesos, la de procesos-resultados, la de factores comunes y la investigación focalizada en la paciente y terapia personalizada. Estas líneas de investigación se diferencian principalmente por las preguntas que realizan respecto del fenómeno psicoterapéutico estudiado. La tabla 1 muestra las preguntas de investigación, los principales tipos de estudios y las principales metodologías empleadas por ellas.

Como podrá notar el lector, la pregunta “¿cómo funciona la terapia narrativa (TN)?” está enmarcada dentro de la línea de investigación de procesos. Es así como el presente trabajo busca responder a esta pregunta por medio de una revisión narrativa de las principales investigaciones de procesos y procesos-resultados que hay publicada sobre la TN hasta la fecha.

Tabla 1. Características de las principales líneas de investigación en psicoterapia.

\begin{tabular}{|c|c|c|c|}
\hline $\begin{array}{c}\text { Línea de } \\
\text { Investigación }\end{array}$ & Pregunta de Investigación & Tipo de Estudios & $\begin{array}{c}\text { Metodología } \\
\text { Privilegiada }\end{array}$ \\
\hline Resultados & ¿Funciona la psicoterapia X? & $\begin{array}{l}\text { - Eficacia (comparación de al menos dos } \\
\text { condiciones de tratamiento a las cuales } \\
\text { los participantes son asignados al azar. } \\
\text { Los tratamientos son manualizados. } \\
\text { Ensayos clínicos aleatorizados) } \\
\text { - Efectividad (comparación de } \\
\text { tratamientos proveídos en las situaciones } \\
\text { naturales del setting clínico) } \\
\text { - Meta-análisis }\end{array}$ & Cuantitativa \\
\hline Procesos & $\begin{array}{l}\text { ¿Cómo funciona la } \\
\text { psicoterapia X? }\end{array}$ & $\begin{array}{l}\text { - Selección de casos representativos } \\
\text { Comparación de agrupaciones de casos } \\
\text { - Caso único }\end{array}$ & $\begin{array}{l}\text { Cualitativa y } \\
\text { cuantitativa }\end{array}$ \\
\hline $\begin{array}{l}\text { Procesos- } \\
\text { resultados }\end{array}$ & $\begin{array}{l}\text { ¿Cómo funciona la } \\
\text { psicoterapia } X \text { para conducir } \\
\text { a Y resultado? }\end{array}$ & $\begin{array}{l}\text { - Selección de casos representativos } \\
\text { Comparación de agrupaciones de casos } \\
\text { - Estudios de mediación y moderación } \\
\text { - Caso único }\end{array}$ & $\begin{array}{l}\text { Cuantitativa y } \\
\text { mixta }\end{array}$ \\
\hline $\begin{array}{l}\text { Factores } \\
\text { Comunes }\end{array}$ & $\begin{array}{l}\text { Si todas las psicoterapias } \\
\text { son igual de eficaces y } \\
\text { efectivas, ¿cuáles son } \\
\text { los factores comunes que } \\
\text { permiten explicar el cambio? }\end{array}$ & $\begin{array}{l}\text { - Estudios de mediación y moderación } \\
\text { - Meta-análisis }\end{array}$ & Cuantitativa \\
\hline $\begin{array}{l}\text { Focalizada en } \\
\text { el paciente } \\
\text { y terapia } \\
\text { personalizada }\end{array}$ & $\begin{array}{l}\text { ¿Está esta psicoterapia } \\
\text { funcionando para esta } \\
\text { persona en este momento? } \\
\text { ¿Qué psicoterapia, proveída } \\
\text { por quién, es la más efectiva } \\
\text { para esta persona, con } \\
\text { este problema específico, } \\
\text { bajo qué conjunto de } \\
\text { circunstancias? }\end{array}$ & $\begin{array}{l}\text { - Efectividad basada en la monitorización } \\
\text { rutinaria del resultado (y en menor medida } \\
\text { de eficacia) } \\
\text { - Selección de casos representativos } \\
\text { Comparación de agrupaciones de casos } \\
\text { - Estudios de mediación y moderación } \\
\text { - Caso único basado en la monitorización } \\
\text { rutinaria del resultado }\end{array}$ & Cuantitativa \\
\hline
\end{tabular}




\section{Antecedentes}

La TN de White y Epston (1993) es un modelo terapéutico que se ha extendido cada vez más en la actualidad (Madigan, 2019). Aún lejos de ser una práctica psicoterapéutica dominante, poco a poco se ha vuelto un modelo cada vez más difundido, conocido y practicado. Desde sus supuestos epistemológicos, ideológicos y éticos (filosofía postmoderna y posestructuralista, meta-teoría construccionista social, ética relacional), la TN se ha manifestado crítica y escéptica respecto del discurso dominante de la investigación en psicoterapia, particularmente respecto de la investigación de resultados y los estudios de eficacia, con sus consecuentes movimientos a favor de los tratamientos empíricamente validados y la práctica de una psicoterapia basada en la evidencia. Siguiendo a Foucault $(1967,1976)$, la TN plantea que estos movimientos, con sus respectivos discursos, serían parte de una aparato socio-político-cultural mayor sustentado en prácticas disciplinarias de vigilancia que promueven la individualización y normalización de la conducta y del fenómeno mental. Desde una perspectiva estructuralista, el sufrimiento y el malestar psicológico son considerados anormales, puesto que las personas tienen dentro de sí determinados rasgos o estructuras psicológicas disfuncionales o deficitarias que les impiden tener una vida 'normal' de acuerdo con ciertos estándares de bienestar. Desde estas ideas, el sufrimiento y el malestar psicológico son concebidos desde una aproximación positivista y psicopatológica(los rasgos psicológicos o las estructuras disfuncionales o deficitarias son objetivables y mensurables) desde las cuales se crean categorías y etiquetas diagnósticas. Para cada diagnóstico se corresponde un tratamiento, el que ha demostrado ser el apropiado a partir de la evidencia empírica de su eficacia. Por el contrario, desde sus asunciones posestructuralistas, la TN plantea que no hay estructuras internas prefijadas respecto de lo humano, sino que toda realidad humana es construida de forma dinámica en el lenguaje, a través de los procesos conversacionales, dialógicos, sociales, políticos e históricos de los que formamos parte. En este sentido, toda investigación respecto de la psicoterapia no es más que un discurso que construye conocimiento y moldea la realidad humana del encuentro psicoterapéutico de acuerdo con ciertos intereses que se han ido configurando política, social y culturalmente a nivel histórico.

Consideramos que estas críticas son válidas. Es más, el discurso de los movimientos a favor de los tratamientos empíricamente validados y la psicoterapia basada en la evidencia margina e invisibiliza otros discursos y otras 'evidencias' respecto de los resultados obtenidos por modelos terapéuticos diversos (no mainstream) y respecto de cómo la psicoterapia funciona independientemente del modelo practicado (factores comunes).

Si asumimos una perspectiva postmoderna (Lyotard, 1994; Vattimo, 1986) lo que surge como característica fundamental del paradigma postmoderno es el multiperspectivismo o la polivocalidad, es decir, la condición de que siempre habrá múltiples perspectivas, múltiples pronunciamientos discursivos o múltiples voces para describir y explicar un mismo fenómeno. En este sentido, Gergen y Warhus 
(2003) caracterizan a las terapias y a los terapeutas postmodernos de la siguiente manera:

Todo conocimiento es una construcción, una construcción que está inmersa en una cultura. Por lo tanto, la terapia eficaz requiere - y es normal que lo haga - discursos múltiples, incluyendo el cultural. (...). Por lo tanto, el terapeuta más completo será el que incluya el discurso romántico, el de la Nueva Era (new age), el del marxismo, el del budismo Zen, entre tantos otros. El terapeuta hábil deberá sentirse cómodo con el lenguaje de la calle, del deporte, del antro, y de Lacan. Porque cada inteligibilidad nueva enriquece el rango y la flexibilidad de la interacción. Este es el pensar del terapeuta construccionista (Gergen y Warhus, 2003, p. 17).

A la lista de discursos que nos dan Gergen y Warhus podemos agregar el discurso de la investigación en psicoterapia, incluido el discurso de la investigación de resultados, los estudios de eficacia y la psicoterapia basada en evidencia. Es así como, a pesar de las críticas de la TN a la investigación en psicoterapia, como terapeutas narrativos es válido preguntarnos si la TN funciona para aquello que dice que funciona. En otras palabras, es válido para quienes practicamos la TN contar con evidencia que permita demostrar que las prácticas terapéuticas que desarrollamos funcionan y ayudan a quienes nos consultan. Es por esto que, antes de preguntarnos cómo funciona la TN, intentaremos responder brevemente a la pregunta de si la TN funciona.

\section{¿Funciona la TN?}

En una revisión anterior respecto de la investigación de resultados sobre la TN (Moggia, 2013), llegábamos a concluir que contábamos con evidencia como para afirmar que la TN sí funciona. En primer lugar, se afirmaba que desde la perspectiva de factores comunes (Duncan, Miller, Wampold y Hubble, 2010; Wampold, 2015), toda psicoterapia eficaz y efectiva cuenta con: 1) el establecimiento de una buena alianza de trabajo entre terapeuta y consultante basada en la colaboración; 2) el desarrollar una explicación viable y adaptativa del malestar del consultante y del curso a seguir para su solución, lo que incrementa las expectativas de mejora del consultante y la confianza en el proceso terapéutico; 3) la preocupación del terapeuta por recibir constante retroalimentación por parte del consultante respecto de la utilidad de lo que se está haciendo y del progreso terapéutico que se va alcanzando con el objetivo de ir adaptando el trabajo a partir de lo que al consultante le es útil y beneficioso; 4) el trabajar considerando lo que al consultante le hace sentido y le es útil desde sus propias fortalezas y recursos; 5) el tener en consideración los factores contextuales o extra-terapéuticos, ya que es en el contexto del consultante donde los cambios y lo que se logra en terapia debe aplicarse para que tenga efectos.

Planteábamos que la TN cumple con las características anteriores al trabajar desde: 1) una postura respetuosa y colaborativa entre terapeuta y consultante basada en la actitud descentrada pero influyente del terapeuta; 2) la externalización, con 
la que el terapeuta invita al consultante a co-construir una explicación viable de su malestar desde su propia historia; 3) la preocupación constante del terapeuta por saber si las conversaciones que se están desarrollando con el consultante le están siendo útiles y tienen sentido para él, para lo que el terapeuta utiliza las preguntas referidas al proceso conversacional (e,g., ¿cómo está yendo esta conversación para ti?); 4) el identificar logros extraordinarios o excepciones al problema, los que constituyen recursos y conocimientos propios del consultante para resolver el problema, los que luego se refuerzan por medio de conversaciones de re-autoría; 5) el involucrar en el proceso terapéutico a otros significativos de la comunidad de la que el consultante participa (e.g.: invitándoles a las sesiones, enviándoles cartas o documentos), velando por el agenciamiento y empoderamiento de la persona fuera de la sesión. La tabla 2 muestra las características de todas las terapias eficaces y efectivas de acuerdo con la perspectiva de factores comunes y cómo la TN cumple con ellas desde sus prácticas.

Tabla 2. Resumen de factores comunes de toda psicoterapia eficaz y efectiva, y prácticas de la terapia narrativa acordes a estos factores.

\section{Factores Comunes Terapia Narrativa}

1. Establecimiento de una buena alianza Postura respetuosa y colaborativa, terapéutica descentrada e influyente

2. El desarrollar una explicación viable y Externalización adaptativa del malestar y del curso a seguir para su solución

3. La preocupación por recibir Preguntas referidas al proceso retroalimentación constante por parte conversacional del consultante

4. El trabajar desde una perspectiva de Trabajo con logros extraordinarios y rerecursos autoría

5. El tener en consideración los factores Involucra a otros significativos de la contextuales comunidad y vela por el agenciamiento de la persona fuera de la sesión

Al cumplir con las características previas, concluíamos que la TN debía ser eficaz y efectiva. Consecuentemente con esta afirmación, se encontraron estudios sobre la efectividad de la TN con buenos resultados en casos de depresión (Vromans y Schweitzer, 2011), encopresis (Silver, Williams, Worthington y Phillips, 1998), trastorno por déficit atencional con hiperactividad (Looyeh, Kamali y Shafieian, 2012), problemas paterno-filiales (Besa, 1994), trastornos de la conducta alimentaria (Weber, Davis y McPhie, 2006), autismo (Cashin, Browne, Bradbury y Mulder, 2013; McGuinty, Armstrong, Nelson y Sheeler, 2012), trastorno por estrés posttraumático (Erbes, Stillman, Wieling, Bera y Leskela, 2014), niños lidiando con fobia social (Yoosefi Looyeh, Kamali, Ghasemi y Tonawanik, 2014), orientación escolary problemas académicos (Beaudoin, Moersch y Evare, 2016), adultos mayores lidiando con problemas de salud mental (Poole, Gardner, Flower y Cooper, 2009). 
Cabe destacar que las características de estos estudios varían mucho de uno a otro, encontrándonos con diversidad de diseños, métodos, muestras y poblaciones, lo que dificulta su comparación y el poder llegar a conclusiones desde la síntesis de resultados. Adicionalmente, la calidad de mucho de ellos no es la mejor, lo que nos impide sacar conclusiones generalizables. La excepción a ello lo constituye el estudio de Vromans y Schweitzer (2011) en el que a través de un benchmarking compara la TN con terapia cognitivo-conductual (TCC) para depresión en adultos. El estudio muestra cómo la TN es al menos tan efectiva como la TCC en la reducción de la sintomatología depresiva al finalizar la terapia y a los tres meses de seguimiento.

Respecto de estudios de eficacia, se encontraron dos. El primero, un ensayo clínico (Lopes, Gonçalves, Fassnacht, Machado y Sousa, 2014; Lopes et al., 2014) en el que se comparó la TN con TCC para depresión en adultos, en el que ambas resultaron igual de eficaces en reducir los síntomas depresivos y los problemas interpersonales tanto al finalizar la terapia como en el seguimiento a los 34 meses. El segundo, corresponde a un diseño experimental (McKian, Rabiei, Bemana y Ramezani, 2019) en el que, mujeres con obesidad fueron asignadas a una de tres condiciones de tratamiento: plan dietético, plan dietético y TN, o grupo control. La condición de plan dietético y TN obtuvo resultados significativamente superiores a las otras dos condiciones en mejorar la imagen corporal y reducir el índice de masa corporal.

$\mathrm{Al}$ revisar los estudios anteriores, no sin limitaciones, podemos concluir que contamos con evidencia como afirmar que la $\mathrm{TN}$ sí funciona para mejorar diversas problemáticas psicológicas. No obstante, se requiere seguir investigando, velando por producir estudios de la suficiente calidad metodológica que permita arribar a resultados generalizables. Sería interesante contar con estudios de la calidad suficiente como para realizar un meta-análisis sobre la efectividad y eficacia de la TN. A partir de aquí, el paso siguiente es preguntarnos cómo funciona la TN. Es con la intención de responder a esta pregunta que a continuación se revisarán los estudios más relevantes de procesos y procesos-resultados sobre la TN.

\section{Los procesos estudiados en TN}

Desde un punto de vista teórico y de forma sintética, el proceso de la TN y su modelo de cambio puede ser explicado de la siguiente manera: la persona, pareja, familia o grupo que consulta acude a terapia contando una historia, la denominada 'historia saturada de problemas', ya que los eventos que se relatan en esta historia sólo dan cuenta de experiencias en las que los problemas que los llevan a consultar están presentes. Esta historia margina otras experiencias de vida no vinculadas a los problemas, que de ser tomadas en cuenta permitirían la construcción de múltiples relatos alternativos desde donde las personas pueden definirse y actuar de forma más satisfactoria para sí mismos y sus otros significativos. La 'historia saturada de problemas' ejerce una relación de poder sobre quienes consultan, puesto que domina la vida de las personas como un única voz o relato a través del cual definirse, 
posicionarse y actuar en el mundo (por eso también se le llama 'relato dominante').

La TN busca que las experiencias que han sido marginadas por el relato dominante y que lo contradicen (denominadas 'logros extraordinarios' o 'excepciones’ al problema), comiencen a sobresalir y a tener voz, de manera de conectarlas dentro de una misma historia, cuya trama nos hablará de unas personas diferentes a las personas saturadas de problemas, personas con conocimientos, posibilidades, sueños y esperanzas para sus vidas, los que antes no eran posibles (Payne, 2002).

Este proceso se va dando a través de las diversas conversaciones que va teniendo el terapeuta con los consultantes a lo largo de las sesiones. Los logros extraordinarios o las excepciones al problema comienzan a ser conectadas en múltiples nuevas historias que dan cuentan de las identidades preferidas de los consultantes (historias que hablan de la persona que les gusta o les gustaría ser). Esto toma lugar a través de las preguntas que va haciendo el terapeuta en la conversación, buscando enriquecer las historias que van emergiendo desde la 'voz' o perspectiva de otros significativos que aparecen en estas historias. El terapeuta asume una postura 'descentrada pero influyente', es decir, por un lado, es consciente de la influencia que él como terapeuta tiene sobre la vida de los consultantes, pero por otro, está atento a que sean los propios consultantes quienes determinen en qué medida y en qué ámbitos de su vida esta influencia tendrá efectos (White, 2002).

Adicionalmente, desde los trabajos de Vygotsky (1980), la conversación terapéutica en TN es concebida como si de la construcción de un andamio se tratase. Un andamio que va de 'lo conocido y familiar' a 'lo que es posible conocer', en el que el terapeuta, a través de sus preguntas, va construyendo los escalones del andamio que le permite a los consultantes atravesar una zona de desarrollo proximal (ZDP) dada por la diferencia entre estas dos posiciones discursivas. Es decir, atravesar un espacio hipotético que habría entre 'lo conocido y familiar', lo que habitualmente se da en la vida de los consultantes, la historia saturada de problemas, y 'lo posible de conocer', las historias alternativas de conocimientos y posibilidades que pueden surgir en la conversación. La distancia que hay entre una historia y otra es la ZDP que el terapeuta ayuda a atravesar a los consultantes por medio de sus preguntas (White, 2016). Las preguntas que va haciendo el terapeuta, en la medida que se van construyendo los escalones del andamio, deben estar justo en el límite superior de la ZDP, ya que si están muy por encima de él resultará una pregunta demasiado inusual para los consultantes y no sabrán cómo responderla. Por el contrario, si la pregunta está por debajo del límite inferior de la ZDP, la pregunta invita a hacer una descripción que no hace más que seguir perpetuando lo ya 'conocido y familiar' (Moggia, en prensa).

Una vez que las historias alternativas han surgido y la historia saturada de problemas se ha deconstruido, los consultantes son invitados a re-narrar estas historias alternativas vinculándolas a sus historias preferidas de identidad (Payne, 2002). El terapeuta va peguntando por los sentimientos, pensamientos y actuaciones de la persona en el pasado, presente y futuro, y desde la perspectiva de otros sig- 
nificativos. A través de este proceso las personas reflexionan sobre su experiencia con mayor perspectiva, construyéndose una identidad más rica que sienta las bases para el afrontamiento de nuevos desafíos futuros.

Cuando ya se ha llegado al final de la terapia, las personas son invitadas a celebrar sus logros con otros significativos que ellos deseen invitar, con el objetivo de que las nuevas historias que han emergido circulen en una comunidad de soporte que les de reconocimiento. Al finalizar, muchas veces los terapeutas preparan documentos que resumen el proceso terapéutico, los logros alcanzados y las habilidades utilizadas para ello, de modo que los consultantes se ‘lleven algo' de la terapia, que podrán leer y consultar en un futuro. Estos documentos pueden ser también escritos por los propios consultantes o también pueden ser diplomas o regalos simbólicos entregados por el terapeuta (White y Epston, 1993).

La descripción anterior acerca del proceso de la TN y su modelo de cambio nos sirve para ilustrar diferentes preguntas de investigación que se pueden realizar al respecto. Por ejemplo: ¿Qué rol cumplen las preguntas y cómo el terapeuta las va formulando? ¿Cómo surgen los logros extraordinarios a lo largo de las sesiones y cuáles son sus características? ¿Qué tipo de historias del consultante emergen y cómo surgen? ¿Cómo se da la colaboración terapéutica a través de la ZDP? ¿Cómo se construye la alianza terapéutica en TN y cómo el terapeuta mantiene una postura descentrada pero influyente? ¿Qué voces o posiciones discursivas surgen en las sesiones y cómo aparecen?, sólo por nombrar algunas. A partir de algunas de estas preguntas podemos clasificar las investigaciones de procesos y procesos-resultados que se han encontrado sobre la TN.

Sin embargo, como sucedía con los estudios de efectividad, los resultados provenientes de los estudios de procesos y procesos-resultados sobre la TN son difíciles de comparar, y por tanto es difícil llegar a conclusiones sintéticas. Esto se debe a la diversidad de temas estudiados, metodologías y diseños empleados. Sin embargo, hay dos programas de investigación que, al estar estructurados como tales, siguen dos líneas concretas de investigación sobre los procesos de cambio en TN, utilizando metodologías y diseños consecuentes y, por tanto, generando resultados comparables. Esto nos permite hacer una revisión exhaustiva de sus publicaciones, comparar los diversos estudios dentro de cada uno de ellos y a partir de ahí, poder sintetizar sus resultados de manera de construir conocimiento acerca de cómo funciona la TN. Por lo tanto, nos focalizaremos en dos de las preguntas planteadas anteriormente: ¿Cómo surgen los logros extraordinarios a lo largo de las sesiones y cuáles son sus características? ¿Cómo se da la colaboración terapéutica a través de la ZDP? La tabla 3 muestra las principales investigaciones halladas que intentan responder a estas preguntas. 


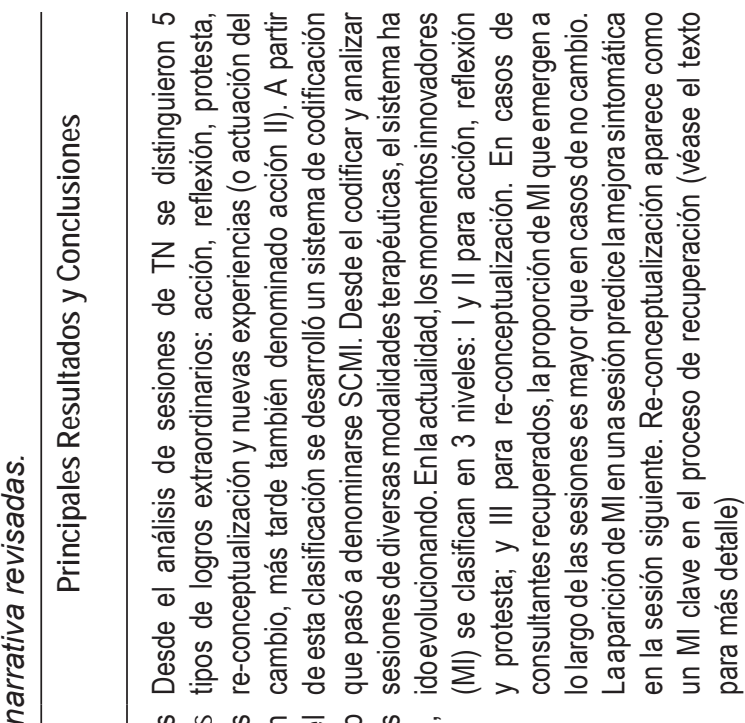

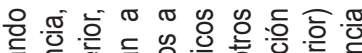

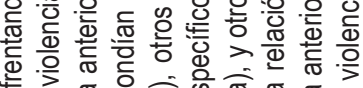

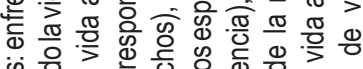

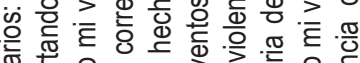

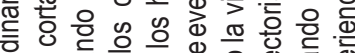

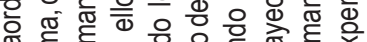

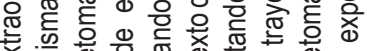
入

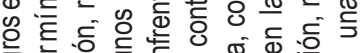

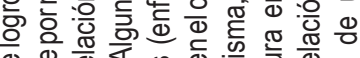

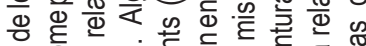

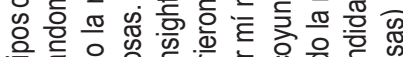

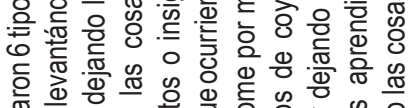
兽

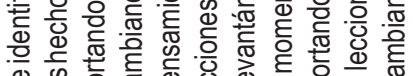
ஸ

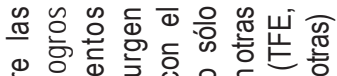

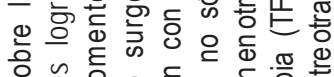

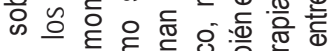

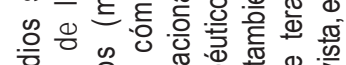

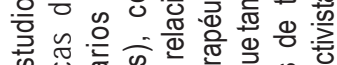

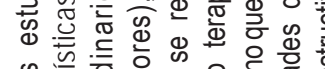

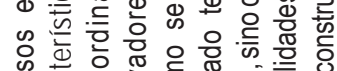

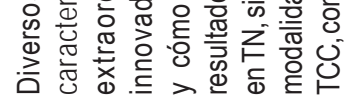

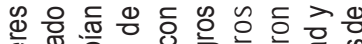
产需 递 $\infty$ क्ष क 응 은

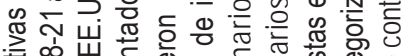

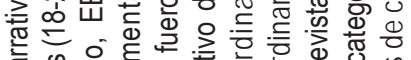
离 号:

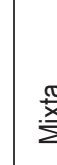

$\stackrel{\frac{\pi}{x}}{\Sigma}$

을

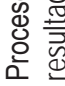

(1)

\section{$>$} 政

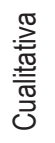

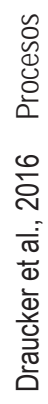

क.

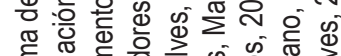

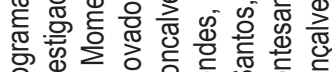

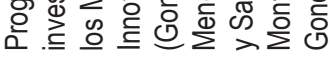




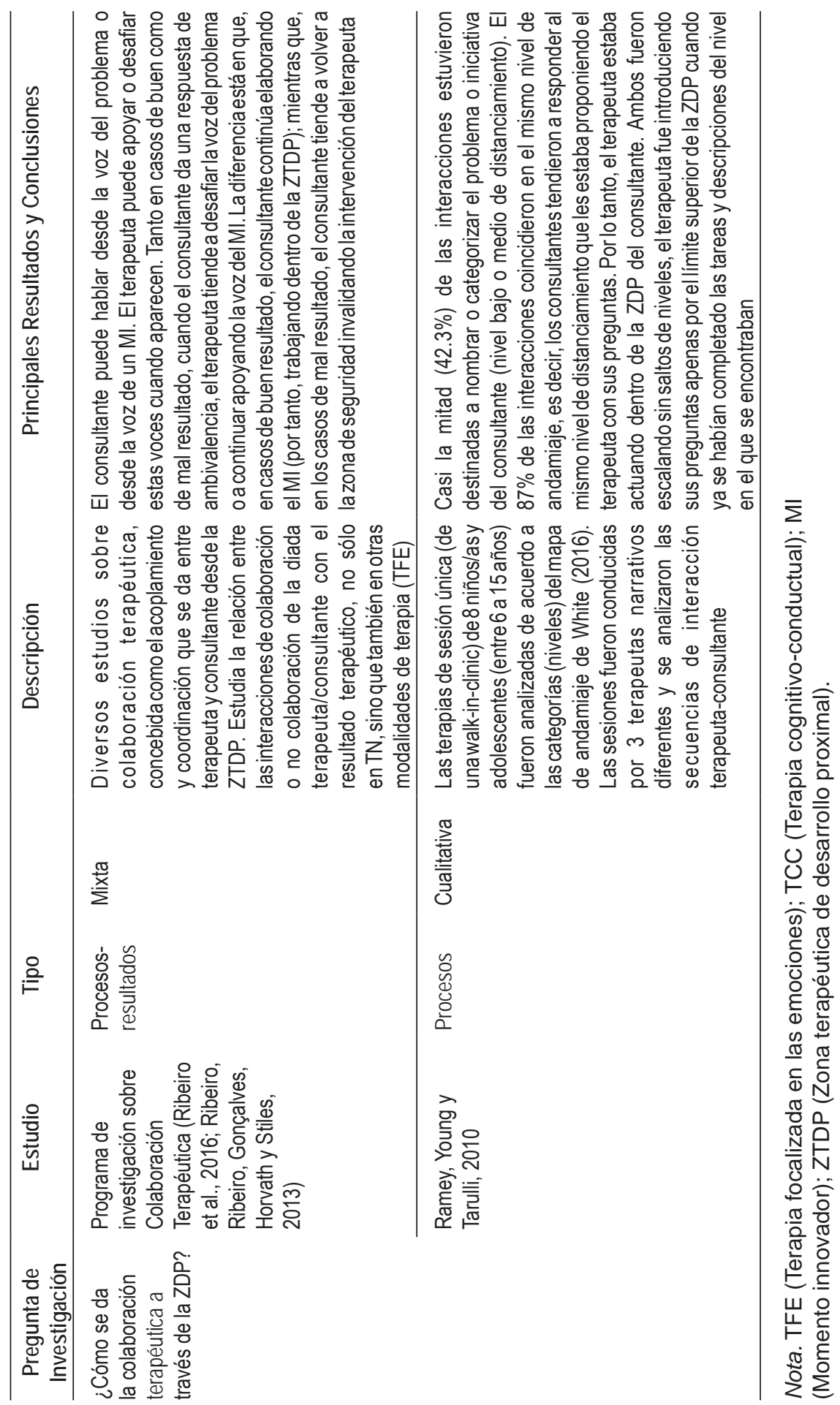




\section{Los logros extraordinarios y el programa de investigación de los momentos innovadores}

El Grupo de Investigación sobre Procesos de Cambio en Psicoterapia, liderado por Miguel Gonçalves de la Universidad de Minho en Portugal, ha desarrollado el programa de investigación sobre los momentos innovadores buscando responder a las siguientes preguntas: ¿Cómo se pueden medir los logros extraordinarios o las excepciones al problema desde un punto de vista empírico? ¿Hay distintos tipos de logros extraordinarios? Si los hay, ¿están relacionados con diferentes patrones de cambio? Si existen diferentes patrones de cambio narrativo, ¿se pueden rastrear 0 identificar a lo largo de las sesiones? ¿Cómo puede evaluarse la relevancia de estos patrones para el proceso de cambio? ¿Cuál es la relación entre cambio narrativo y mejora sintomática? (Montesano, Oliveira y Gonçalves, 2017).

A partir de 2004, el equipo de Gonçalves comenzó a trabajar para la identificación de logros extraordinarios en sesiones de TN por medio de análisis de contenido. Definieron operacionalmente los logros extraordinarios como cualquier pensamiento, sentimiento o acción del consultante que no fuese predicho por las reglas o mandatos del relato dominante y que fuese diferente o novedoso respecto de la forma en que el consultante se narraba usualmente a sí mismo (Gonçalves, Matos y Santos, 2009). En sesiones de TN con mujeres que habían sufrido violencia de pareja (Matos y Gonçalves, 2004), lograron identificar cinco tipos de logros extraordinarios: acción (cualquier acción emprendida en contra del problema o a favor del proyecto de cambio), reflexión (nuevas comprensiones, entendimientos, sentimientos o perspectivas acerca del problema o que desafían las prescripciones culturales que facilitan el desarrollo de la narrativa problemática), protesta (críticas en contra del problema en donde la persona toma un nuevo posicionamiento o reafirma sus propias necesidades y derechos), re-conceptualización (recuento de un proceso de cambio en donde la persona dice qué es diferente en ella y cómo ha logrado producir esa diferencia) y nuevas experiencias (o actuación del cambio, más tarde también denominado acción II, nuevas acciones que la persona está emprendiendo a partir del proceso de cambio o que desea emprender; véase la tabla 4 para más detalles). Adicionalmente, observaron que en casos de buen resultado terapéutico (casos recuperados) la proporción de logros extraordinarios era mucho mayor que en casos de mal resultado terapéutico (casos de no cambio), y que, además, re-conceptualización sólo aparecía en los casos de buen resultado.

Los hallazgos anteriores los llevaron a hipotetizar que re-conceptualización sería un marcador clave dentro del proceso de recuperación. Re-conceptualización representaría una meta-posición del 'yo', la que introduce un contraste entre el 'yo del pasado' y el 'yo actual', permitiendo un descentramiento de la persona al ser capaz de explicar, desde una posición meta-reflexiva, cómo ha logrado hacer esta transición. En este sentido, re-conceptualización trasciende la dicotomía semiótica problema vs. no-problema al ir más allá del mero reemplazo de una narrativa por 
otra (la dominante por una alternativa), dando cuenta de un completo proceso de transformación llevado a cabo por la persona desde el ir cambiando sus formas de pensar, sentir y actuar, para dar paso a un 'nuevo yo' (Gonçalves et al., 2009). Esto implica que para llegar al marcador de re-conceptualización, primero deben darse otro tipo de logros extraordinarios de menor nivel de complejidad o abstracción narrativa como lo son acción, reflexión y protesta. Consecuentemente con esta idea, el equipo de Gonçalves hipotetizó que, en casos de buen resultado terapéutico, durante las fases iniciales de la terapia, debían aparecer logros extraordinarios de bajo nivel de complejidad como acción, reflexión y protesta, para luego, en fases intermedias, comenzar a aparecer re-conceptualización. A partir de la emergencia de re-conceptualización, nuevos logros extraordinarios, esta vez de mayor complejidad, del tipo nuevas experiencias, reflexión y protesta comenzarían a surgir, dando paso a la emergencia de nuevos marcadores de re-conceptualización. Posteriormente, en las fases finales de la terapia, la narrativa dominante habría dejado de prevalecer, dando paso a múltiples narrativas alternativas de identidad desde donde la persona podría posicionarse y definirse. Por el contrario, en casos de mal resultado terapéutico, re-conceptualización estaría ausente en las fases intermedias de la terapia, es decir, en las fases iniciales aparecerían los marcadores de acción, reflexión y protesta de bajo nivel de complejidad, posteriormente en las fases intermedias seguirían apareciendo estos mismos marcadores en ausencia de reconceptualización, para finalmente, en las fases finales de la terapia, la narrativa dominante continuar prevaleciendo.

En sus trabajos posteriores, el equipo de Gonçalves no sólo quiso poner a prueba estas hipótesis, sino que además quiso averiguar si en sesiones de terapia para otro tipo de problema que no fuese violencia de pareja, los logros extraordinarios que se habían identificado podían hallarse también. Adicionalmente, quisieron averiguar si en otro tipo de terapia que no fuese TN, también aparecerían este tipo de logros extraordinarios. A partir de aquí, formalizaron las categorías de logros extraordinarios que habían encontrado como un sistema de codificación que podía utilizarse para rastrear novedad en las narrativas de los consultantes a lo largo de la terapia, independientemente del modelo terapéutico y, por tanto, estudiar procesos de cambio de forma transteórica. Había surgido el Sistema de Codificación de Momentos Innovadores (SCMI).

Para diferenciar el nivel de complejidad o de abstracción narrativa de los momentos innovadores (MI), se definieron MI de nivel I, II y III (Fernández-Navarro, Ribeiro, Soylemez y Gonçalves, 2019). Los de nivel I corresponden a innovaciones en la narrativa del consultante que crean distancia del problema. Pueden aparecer en forma de acciones, reflexiones (sentimientos o pensamientos) o protestas (críticas) en contra del problema. Los de nivel II son innovaciones en la narrativa del consultante que están centradas en el proceso de cambio, haciendo referencia a nuevos objetivos, experiencias, actividades o proyectos que ya están ocurriendo en el presente o que están por ocurrir en un futuro, sin hacer referencia al proble- 
ma o no necesariamente relacionados con el problema. Pueden tomar la forma de acciones, reflexiones o protestas. Por último, los de nivel III, re-conceptualización, representan la meta-posición de la que se habló anteriormente, la que da cuenta de qué ha cambiado en la vida del consultante (contraste del sí-mismo) y de cómo se ha logrado ese cambio (descripción del proceso de cambio). La tabla 4 muestra la definición de cada uno de los MI junto con un ejemplo.

Tabla 4. Momentos de innovación con ejemplos.

\begin{tabular}{|c|c|c|c|}
\hline Tipos de MI & Subtipos & Definición & $\begin{array}{c}\text { Ejemplos } \\
\text { (Narrativa problemática: depresión) }\end{array}$ \\
\hline \multirow{3}{*}{$\begin{array}{l}\text { MI de } \\
\text { nivel I } \\
\text { (Crean } \\
\text { distancia } \\
\text { del pro- } \\
\text { blema) }\end{array}$} & Acción I & $\begin{array}{l}\text { Acciones realizadas } \\
\text { e intentadas para } \\
\text { enfrentar el proble- } \\
\text { ma }\end{array}$ & C: ¡Ayer fui al cine por primera vez en meses! \\
\hline & Reflexión I & $\begin{array}{l}\text { Nuevas compren- } \\
\text { siones acerca del } \\
\text { problema }\end{array}$ & $\begin{array}{l}\text { C: Me doy cuenta de que lo que estaba hacien- } \\
\text { do no era humanamente posible, me estaba } \\
\text { presionando a mí misma y no me daba tiempo } \\
\text { libre, uh, para mí... y es más natural y más sano } \\
\text { permitir algunas de estas actividades extras... }\end{array}$ \\
\hline & Protesta I & $\begin{array}{l}\text { Oponerse al } \\
\text { problema y a sus } \\
\text { supuestos }\end{array}$ & $\begin{array}{l}\text { C: ¿En qué me estoy convirtiendo finalmente? } \\
\text { ¿En qué me estoy metiendo? ¿¡Me voy a } \\
\text { estancar aquí!? }\end{array}$ \\
\hline \multirow[t]{3}{*}{$\begin{array}{l}\text { MI de } \\
\text { nivel II } \\
\text { (Centra- } \\
\text { dos en el } \\
\text { cambio) }\end{array}$} & $\begin{array}{l}\text { Actuación } \\
\text { del cambio } \\
\text { (Acción II) }\end{array}$ & $\begin{array}{l}\text { Generalización de } \\
\text { buenos resultados } \\
\text { hacia el futuro y } \\
\text { otras dimensiones } \\
\text { de la vida (reali- } \\
\text { zados o acciones } \\
\text { proyectadas) }\end{array}$ & $\begin{array}{l}\text { T: ¡Pareces tener tantos proyectos para el futuro } \\
\text { a partir de ahora! } \\
\text { C: Sí, tienes razón. Quiero hacer todas las cosas } \\
\text { que me eran imposibles de hacer cuando estaba } \\
\text { dominado por la depresión. Quiero trabajar de } \\
\text { nuevo y tener el tiempo para disfrutar de mi vida } \\
\text { con mis hijos. Quiero tener amigos nuevamente. } \\
\text { La pérdida de todas las amistades del pasado } \\
\text { es algo que aún me duele profundamente. }\end{array}$ \\
\hline & $\begin{array}{l}\text { Reflexión } \\
\text { II }\end{array}$ & $\begin{array}{l}\text { Contraste del } \\
\text { sí-mismo (¿qué ha } \\
\text { cambiado?) } \\
\text { O } \\
\text { Proceso de transfor- } \\
\text { mación (¿cómo/por } \\
\text { qué ha ocurrido el } \\
\text { cambio?) }\end{array}$ & $\begin{array}{l}\text { C: Me siento fuerte y positivo. Está bien pregun- } \\
\text { tarse por estas cosas [sus necesidades], es una } \\
\text { nueva parte de mí, así que no voy a derrumbar. }\end{array}$ \\
\hline & Protesta II & $\begin{array}{l}\text { Asertividad y empo- } \\
\text { deramiento }\end{array}$ & $\begin{array}{l}\text { C: Soy un adulto y soy responsable de mi vida, } \\
\text { y quiero reconocer estos sentimientosy jme voy } \\
\text { a permitir sacarlos fuera! Quiero experimentar } \\
\text { la vida, quiero crecer y se siente bien estar a } \\
\text { cargo de mi propia vida. }\end{array}$ \\
\hline
\end{tabular}




\begin{tabular}{|c|c|c|c|}
\hline Tipos de MI & Subtipos & Definición & $\begin{array}{c}\text { Ejemplos } \\
\text { (Narrativa problemática: depresión) }\end{array}$ \\
\hline $\begin{array}{l}\text { MI de } \\
\text { nivel III } \\
\text { (Qué ha } \\
\text { cambiado } \\
\text { y cómo } \\
\text { se ha } \\
\text { logrado) }\end{array}$ & $\begin{array}{l}\text { Re- } \\
\text { concep- } \\
\text { tualización }\end{array}$ & $\begin{array}{l}\text { Momento de } \\
\text { distanciamiento } \\
\text { de la experiencia } \\
\text { (meta-posición) don- } \\
\text { de el sí-mismo es } \\
\text { reposicionado fuera } \\
\text { de la experiencia } \\
\text { problemática } \\
\text { Y existe una } \\
\text { comprensión del } \\
\text { proceso involucrado } \\
\text { en esta transforma- } \\
\text { ción }\end{array}$ & $\begin{array}{l}\text { C: Actualmente me siento diferente. No me } \\
\text { preocupa lo que otros piensen acerca de lo } \\
\text { que estoy diciendo. Descubrí que necesito } \\
\text { respetar mis necesidades y opiniones, incluso } \\
\text { si hay personas que están en desacuerdo. } \\
\text { Antes de protegerme de discordar con otros } \\
\text { estaba siempre en conflicto conmigo mismo - } \\
\text { pensando una cosa, diciendo otra. ¿Qué hace } \\
\text { que el desacuerdo con otros sea peor que esta } \\
\text { lucha interna? }\end{array}$ \\
\hline
\end{tabular}

Nota. MI (Momentos innovadores). Adaptada de Oliveira, Gonçalves, Braga y Ribeiro (2016).

En la actualidad, más de 600 sesiones de terapia han sido codificadas utilizando el SCMI (Montesano et al., 2017), las que incluyen: TN para casos de violencia de pareja, TN para depresión, terapia focalizada en las emociones (TFE), terapia constructivista para el duelo, TCC, terapia centrada en el cliente, entre otras. La mayoría de los estudios siguen el mismo diseño de procesos-resultados en el que se comparan los patrones de cambio narrativo obtenidos por casos de buen y mal resultado terapéutico (casos recuperados y de no cambio, respectivamente).

En términos generales, los resultados obtenidos por estos estudios tienden a apoyar las hipótesis iniciales del equipo de Gonçalves. Esto es, en casos de buen resultado terapéutico existe una tendencia de progresión en la proporción de MI en las narrativas del consultante a medida que la terapia avanza. Los MI de nivel I emergen desde el inicio de la terapia, en cambio los de nivel II y III tienden a aparecer en las fases intermedias, continuando hasta el final. Los casos de mal resultado terapéutico tienden a presentar una proporción significativamente menor de MI al compararlos con los casos de buen resultado. Los MI de nivel I emergen en las sesiones sin una clara tendencia de progresión a lo largo de la terapia. Los MI de nivel II y III están ausentes o aparecen en una proporción significativamente baja al compararlos con casos de buen resultado terapéutico.

Finalmente, lo que el equipo de Gonçalves ha continuado explorando es la relación entre MI y mejora sintomática (Gonçalves, Ribeiro, Silva, Mendes y Sousa, 2015). Para esto, analizaron 10 casos de TN para depresión (18 sesiones en promedio cada uno) y se utilizaron modelos lineales jerárquicos para ver si la aparición de MI en una sesión predice o explica una mejora sintomática a la sesión siguiente, o, por el contrario, si una mejora sintomática precede el surgimiento de MI. Los resultados 
indicaron que los MI son mejores predictores de la mejora sintomática a la sesión siguiente que a la inversa. Adicionalmente, a través del mismo diseño quisieron probar la relación entre re-conceptualización y mejora sintomática(Fernández-Navarro et al., 2018). Esta vez, analizaron 16 casos de TN para depresión (19 sesiones en promedio cada caso) obteniendo los siguientes resultados: re-conceptualización obtuvo una frecuencia menor de aparición a lo largo de la sesiones que reflexión II (o que sus dos componentes por separado: contraste del sí-mismo y descripción del proceso de cambio), pero re-conceptualización resultó ser un mejor predictor de la mejora sintomática a la sesión siguiente que reflexión II. Además, la aparición de re-conceptualización en una sesión es mejor predictor de la mejora sintomática a la sesión siguiente que a la inversa. Estos resultados apoyan la hipótesis de cómo surgen los MI en casos recuperados y que re-conceptualización es clave en este proceso. Así mismo, el cambio narrativo (o el surgimiento de logros extraordinarios) no es un epifenómeno de la mejora sintomática, sino que las innovaciones narrativas serían capaces de promover la reducción de síntomas, al menos en TN para depresión (Montesano et al., 2017).

\section{La ambivalencia}

Otro de los fenómenos estudiados por el grupo de Gonçalves es el de ambivalencia. Ellos se dieron cuenta que en TN cuando un consultante produce un MI y el terapeuta interviene preguntando acerca de él, el consultante tiene dos posibilidades: amplificarlo o atenuarlo. El consultante lo amplifica cuando continúa desarrollando y elaborando la narrativa ligada al MI de acuerdo con las preguntas del terapeuta, profundizando en él y aceptando su valor potencial de novedad e innovación. Por el contrario, se produce una atenuación del MI cuando a partir de las preguntas del terapeuta, el consultante se contradice, se reafirma en la narrativa problemática, re-atribuye o trivializa la experiencia restándole su valor potencial de novedad e innovación. A este comportamiento que puede ser identificado y rastreado a lo largo de las sesiones, se la ha denominado Marcador de Retorno al Problema (MRP) y al sistema de codificación diseñado para su identificación, Sistema de Codificación de la Ambivalencia (SCA, Gonçalves et al., 2011).

Para el equipo de Gonçalves, de lo que estaría dando cuenta un MRP es de un fenómeno de ambivalencia (Braga et al., 2018). Desde una perspectiva dialógica, la aparición de un MI estaría desafiando el marco experiencial y de entendimiento de la persona, generando una sensación de riesgo e incertidumbre. Cuando la incertidumbre se vuelve amenazante, para lidiar con ella la persona utilizaría una estrategia semiótica caracterizada por la atenuación de la novedad, sus significados e implicaciones, retornando rápidamente a la narrativa dominante. En este sentido, una voz dominante y una no dominante (o innovadora) establecen una relación circular de mutua alimentación, bloqueando el desarrollo o cambio personal (Ribeiro y Gonçalves, 2011). Por ejemplo, una persona que consulta por problemas de ansiedad social y la imposibilidad de decir que no a los demás en 
diversas situaciones. Su narrativa dominante podría ser caracterizada como "Por lo general soy muy sumiso.” A lo largo de las sesiones, podría surgir un MI del tipo: "A veces me digo a mí mismo: 'No quiero hacer lo que $\mathrm{X}$ persona me pidió”" (Protesta I), para luego surgir un MRP: "Pero luego me siento una persona egoísta si no lo hago", reafirmándose la narrativa dominante: "He sido sumiso toda mi vida, es simplemente la manera que soy” (Gonçalves et al., 2011).

En casos de TN para mujeres víctimas de violencia de pareja (Gonçalves et al., 2011), la proporción de MRP fue mucho más alta en casos de no cambio que en casos recuperados. Esto indicaría que el mal resultado terapéutico estaría vinculado con un retorno sistemático a la narrativa dominante por parte del consultante luego de producir una innovación narrativa. Resultados similares fueron obtenidos en casos de TN para depresión (Ribeiro, Gonçalves, Silva, Brás y Sousa, 2015). Desde un punto de vista longitudinal, ambos grupos, recuperados y de no cambio, presentaron la misma proporción de MRP en las sesiones iniciales. Sin embargo, los MRP tendieron a disminuir progresivamente a lo largo de las sesiones en casos recuperados, mientras que en casos de no cambio, si bien, también los MRP tendieron a disminuir a lo largo del proceso terapéutico, su disminución fue significativamente menor que la de los casos recuperados. Al final del tratamiento, los casos de no cambio presentaron una proporción mucho mayor de MRP que lo casos recuperados.

Los resultados anteriores sugieren que los MRP como marcadores de ambivalencia son un fenómeno común tanto en casos de buen como de mal resultado terapéutico, probablemente dando cuenta de la sensación de riesgo e incertidumbre que el cambio puede provocar. En este sentido, la ambivalencia puede ser considerada un mecanismo de protección utilizado por el consultante para lidiar con el riesgo e incertidumbre producida por la novedad implicada en la ruptura de sus mecanismos de funcionamiento habituales.

Considerando que la ambivalencia está presente desde un inicio en los dos tipos de casos analizados y que la disminución de ésta a lo largo de la terapia es mucho mayor en casos recuperados que en los casos de no cambio, la diferencia entre ambos grupos radicaría en que los casos recuperados desarrollarían una estrategia para su resolución a lo largo del proceso terapéutico. Para estudiar esta hipótesis, el equipo de Gonçalves desarrolló el Sistema de Codificación de Resolución de la Ambivalencia (SCRA, Gonçalves et al., 2017).

El SCRA considera que habría dos mecanismos utilizados por el consultante para resolver la ambivalencia a lo largo del proceso terapéutico: dominación y negociación. La resolución por dominación implicaría que la voz innovadora pasa a dominar la vida de la persona subyugando a la que anteriormente fue la voz del relato dominante. Consiste en una inversión de roles. En cambio, en la resolución por negociación, las voces o posiciones conflictuadas parecen comunicarse respetuosamente la una con la otra, promoviendo un fluir dinámico entre los opuestos, más que la dominación de una por sobre la otra (Oliveira, Gonçalves, Braga y 
Ribeiro, 2016). En otras palabras, hay una elaboración conjunta de significados entre ambas posiciones, creando una semántica que las trasciende.

En el principal estudio en el que se ha utilizado el SCRA se han analizado 10 casos de TN, 6 casos de TCC y 6 casos de TFE para depresión (Braga et al., 2018). En los tres tipos de terapia, en casos recuperados, negociación obtuvo una proporción mucho mayor que dominación. Por el contrario, en casos de no cambio, dominación fue mucho más frecuente que en los casos recuperados. En estos últimos, a lo largo de la terapia, la proporción de dominación fue disminuyendo y la de negociación fue aumentando. En cambio, en casos de no cambio, la proporción de dominación tendió a aumentar ligeramente a lo largo del proceso terapéutico y negociación se mantuvo prácticamente constante a niveles bajos (casi ausente). Es interesante que los casos recuperados obtuvieron proporciones altas de negociación desde el inicio de la terapia, al compararlos con casos de no cambio, y que ambos grupos iniciaron la terapia con niveles similares de dominación. Esto puede sugerir que ambos grupos entraron a la terapia en diferentes estadios de cambio y que el desarrollo de estrategias de negociación desde el inicio de la terapia representaría un factor importante para el éxito terapéutico.

Por último, al igual que con los MI, se analizó el valor predictivo de los MRP sobre la reducción sintomática (Braga, Ribeiro, Sousa y Gonçalves, 2019). Para ello se seleccionaron 10 casos de TN y 6 casos de TCC para depresión. Los resultaron indicaron que existe una relación bidireccional entre MRP y síntomas, es decir, por un lado, a menor proporción de MRP en una sesión, menor sintomatología en la sesión siguiente; y por otro, a mayor sintomatología en una sesión, mayor ambivalencia en la sesión siguiente.

Para sintetizar, de acuerdo al programa de investigación de Gonçalves, existen al menos siete tipos de MI que pueden ser clasificados de acuerdo a su nivel de complejidad o abstracción narrativa (véase tabla 4). Los MI de mayor complejidad tienden a aparecer más frecuentemente en los casos de buen resultado terapéutico a partir de las fases intermedias de la terapia, destacando el valor de reconceptualización como un proceso narrativo clave en la recuperación. De acuerdo a lo planteado por la TN, es la aparición y elaboración de logros extraordinarios (MI) lo que contribuye a la mejora sintomática a lo largo del proceso terapéutico. La ambivalencia es un fenómeno que puede aparecer en los casos tanto de buen como de mal resultado terapéutico. Los casos de buen resultado tienden a resolverla empleando principalmente la estrategia de negociación. Sin embargo, para llegar al proceso de negociación, al parecer, primero deben darse resoluciones por dominación (Oliveira et al., 2016).

\section{Andamiajes, zona de desarrollo proximal y el programa de investigación sobre colaboración terapéutica}

El Grupo de Investigación sobre Relación Terapéutica, liderado por Eugenia Ribeiro, también de la Universidad de Minho en Portugal, ha desarrollado 
el programa de investigación sobre colaboración terapéutica. El programa busca estudiar las conductas de colaboración o no colaboración que se dan entre terapeuta y consultante a lo largo del proceso terapéutico como un aspecto fundamental de la alianza de trabajo. Para ello, han desarrollado el Sistema de Codificación de Colaboración Terapéutica (SCCT, Ribeiro, Ribeiro, Gonçalves, Horvath y Stiles, 2013). Este sistema permite evaluar cómo se va dando la coordinación entre las propuestas e intervenciones del terapeuta y las respuestas del consultante momento a momento en la sesión. En otras palabras, evalúa si las intervenciones del terapeuta responden o no a las necesidades de cambio del consultante, y si las respuestas del consultante se corresponden o no a las propuestas o desafíos del terapeuta. Basado en el concepto de ZDP, el sistema asume que el cambio del consultante ocurre dentro de una zona terapéutica de desarrollo proximal (ZTDP), y que la colaboración terapéutica resulta de un esfuerzo conjunto del terapeuta y del consultante para trabajar dentro de esta zona.

El SCCT define la ZTDP como el espacio entre la capacidad actual del consultante de acomodar un MI (amplificarlo y aceptar su valor potencial de novedad e innovación), usualmente muy limitada cuando el consultante busca ayuda debido a la rigidez del relato dominante, y la capacidad potencial que puede ser alcanzada en colaboración con el terapeuta.

El SCCT supone que el consultante siempre está hablando, o bien, desde la voz del problema, o desde la voz de un MI. A su vez, el terapeuta puede apoyar o desafiar cada una de estas voces cuando aparecen. Por otra parte, el consultante puede validar o invalidar las intervenciones del terapeuta. De esta manera, lo que ocurriría a partir de los distintos niveles de la ZTDP es lo siguiente:

1) Debajo del límite inferior. El terapeuta interviene sobre algo ya resuelto o irrelevante para el problema actual, el consultante experimenta un riesgo mínimo mostrando desinterés.

2) Límite inferior. El consultante acepta la perspectiva del terapeuta, pero luego toma la perspectiva opuesta intercambiando comportamientos de validación e invalidación. El consultante puede saltar espontáneamente por sobre el nivel que le está proponiendo el terapeuta y desplazarse hacia una zona de riesgo y experimentar ambivalencia.

3) Cerca del nivel actual del consultante. El terapeuta apoya la voz del problema (el relato dominante, por ejemplo, pidiendo más información para entender el problema y la perspectiva del consultante al respecto), el consultante experimenta una sensación de seguridad.

4) Cerca del nivel potencial del consultante. El terapeuta apoya la voz del MI y el consultante lo amplifica. El consultante experimenta un riesgo tolerable.

5) Límite superior. El consultante atenúa el MI ya que estaría experimentando riesgo alto y por tanto buscaría volver a su zona de seguridad. El comportamiento que aparece es el de ambivalencia (MRP). 
6) Sobre el límite superior. El consultante experimenta riesgo intolerable, invalidando las intervenciones del terapeuta. El consultante se muestra defensivo, cambia el tema o no entiende al terapeuta.

En un estudio de caso (Ribeiro et al., 2014) consistente en una mujer de 47 años que recibió 15 sesiones de TN por problemas de violencia de pareja, la que finalmente mostró un mal resultado terapéutico, se pudo observar que la mayoría de las apariciones de MRP estaban precedidas por una intervención de desafío de la voz del problema por parte de la terapeuta. Cuando la terapeuta apoyaba un MI, sólo algunas veces aparecieron MRP. Cuando la terapeuta apoyaba la voz del problema, nunca aparecieron MRP. A su vez, cuando apareció un MRP, la terapeuta tendió a continuar con intervenciones de desafío, provocando consecutivamente una respuesta de invalidación por parte de la consultante. Sin embargo, cuando la terapeuta validaba la voz del problema luego de un MRP, la consultante validaba a la terapeuta. No obstante, la consultante mostró mínimas respuestas de validación de la terapeuta a lo largo de la terapia y sólo muy pocas veces respondió en el nivel que se le estaba proponiendo, y por tanto, elaborando un MI.

Otro estudio (Ribeiro et al., 2016) comparó los procesos de colaboración en dos casos de TN para depresión tratados por el mismo terapeuta, buen (hombre de 22 años) y mal (mujer de 30 años) resultado terapéutico. En ambos casos, los MRP surgieron cuando el terapeuta apoyaba las voces de innovación narrativa (trabajando en el límite superior de la ZTDP) o desafiaba la voz del problema. El terapeuta mostró proporciones similares de intervenciones de desafío y apoyo de la voz problemática en ambos casos. En el caso de buen resultado, cuando el terapeuta respondía a los MRP apoyando la voz del problema, el consultante se desplazaba por sobre el nivel propuesto elaborando el MI precedente. Cuando el terapeuta respondía desafiando la voz del problema, el consultante validaba la intervención del terapeuta, para posteriormente continuar elaborando el MI (respondía en el nivel propuesto). En el caso de mal resultado, cuando el terapeuta apoyaba la voz dominante después de un MRP, la mayoría de las veces, la consultante respondía en el nivel propuesto. Cuando el terapeuta desafiaba la voz dominante después de un MRP, la consultante tendía a validar mínimamente al terapeuta, sin elaborar posteriormente el MI.

Finalmente, los procesos de colaboración fueron analizados en siete casos (6 mujeres, entre 19 y 43 años) de abandono de TN para depresión tratados por el mismo terapeuta (Pinto, Sousa, Pinheiro, Freitas y Ribeiro, 2018). En general, el terapeuta tendió a exhibir una alta proporción de desafío de la voz problemática en todos los casos, la que tendió a aumentar progresivamente a lo largo de las sesiones. A su vez, las respuestas más frecuentes de los consultantes fueron respuestas desde su zona de seguridad (nivel 3) o de retorno a la misma (ambivalencia). De esta manera, cuando el terapeuta apoyaba la voz del problema los consultantes respondían desde este nivel, mientras que, cuando apoyaba la voz de los MI, los consultantes respondían con un MRP. Adicionalmente, cuando el terapeuta desafiaba la voz del 
problema, los consultantes también tendían a exhibir ambivalencia. Las respuestas más frecuentes del terapeuta ante las respuestas de los consultantes continuaron siendo respuestas de desafío de la voz problemática, incluso ante respuestas de desinterés por parte de los consultantes.

Los resultados anteriores enseñan que los casos de mal resultado terapéutico (y de abandono) tienden a responder frente a las intervenciones que desafían el relato dominante, o frente a las que apoyan las innovaciones narrativas, retornado a su zona de seguridad, es decir, produciendo respuestas de ambivalencia. A su vez, ante la respuesta de ambivalencia, los terapeutas continúan desafiando la voz del problema, provocando respuestas de invalidación de la intervención por parte del consultante. En este contexto, la sensación de riesgo o amenaza percibida por el consultante podría precipitar la decisión de abandonar la terapia.

Lo anterior sugiere que parte de la ambivalencia que surge durante el proceso terapéutico, en los casos de mal resultado, tendría a ser elicitada por el terapeuta al persistir en trabajar en el límite superior de la ZTDP. Es interesante que cuando los terapeutas responden a un MRP con una intervención de desafío de la voz problemática, el diálogo terapéutico suele salirse de la ZTDP, produciendo una escalada en la sensación de riesgo del consultante. En este sentido, los terapeutas no estarían siendo capaces de restaurar la colaboración terapéutica (trabajar dentro de la ZTDP) luego de producirse estos episodios de pérdida de calidad en la misma.

Por el contrario, en los casos de buen resultado terapéutico, aunque los terapeutas suelen responder de igual forma que en los casos de mal resultado ante la aparición de ambivalencia (desafiando la voz dominante), el consultante tiende a validar la intervención del terapeuta respondiendo en el nivel propuesto o yendo por sobre él para continuar elaborando el MI. Esto sugiere que, en los casos de mal resultado, habría un problema en la sensibilidad de los terapeutas para adaptar y corregir sus intervenciones de acuerdo con las interacciones que van desarrollando con sus consultantes desde el nivel de la ZTDP en la que se encuentran.

\section{Conclusiones: ¿Cómo funciona la TN?}

Los resultados de los estudios revisados permiten concluir que la TN funcionaría de acuerdo con el modelo de cambio que ella propone. Esto es, promoviendo la emergencia de logros extraordinarios (o MI) como una forma de flexibilizar el relato dominante y co-construir alternativas a éste en términos de nuevas formas de pensar, sentir y actuar. La emergencia de logros extraordinarios a lo largo del proceso terapéutico estaría relacionada con la mejora sintomática y la obtención de un buen resultado terapéutico, sobre todo, la aparición de logros extraordinarios de mayor complejidad a partir de las fases intermedias de la terapia. En este sentido, re-conceptualización constituye un proceso clave del proceso de cambio, representando una meta-posición que permitiría la transición entre el antes y el después en la vida (identidad) del consultante.

Comolo plantea White, la colaboración entre terapeuta y consultante dependería 
de la colaboración entre ambos desde de la ZDP. Intervenciones terapéuticas dentro de la ZDP favorecería la elaboración y asimilación de los logros extraordinarios. Lo contrario promovería ambivalencia. Una alta ambivalencia o la no resolución de la misma estaría asociada a casos de no cambio o abandono. Adicionalmente, la resolución de la ambivalencia a través de negociación estaría relacionada con un mejor resultado terapéutico que a una resolución por dominación. Por último, se requiere mayor investigación sobre colaboración terapéutica en TN para comprender el rol que juega la sensibilidad del terapeuta en corregir y adaptar sus intervenciones de acuerdo al nivel de la ZDP en la que se encuentra el consultante y de esta forma co-construir un andamiaje favorable al proceso de cambio.

\section{Discusión}

El objetivo del presente trabajo era responder a la pregunta “¿cómo funciona la TN?”. Para esto, primero se intentó responder a la pregunta de si la TN funciona. Asumiendo la perspectiva de factores comunes, se concluía que la TN debía ser eficaz y efectiva al cumplir con las características de toda psicoterapia eficaz y efectiva desde sus prácticas. Para respaldar esta afirmación, se revisaron las principales investigaciones de resultados que hay sobre la TN concluyendo que, si bien existe evidencia como para afirmar que la TN sí funciona, aún falta mucho por hacer para mejorar la calidad de los estudios. Desde este punto, se realizó una revisión narrativa de las principales investigaciones de procesos y procesos-resultados que hay publicadas con el objetivo de responder a la pregunta inicial.

Los estudios de procesos y procesos-resultados encontrados son diversos, difiriendo en temas, diseños y metodologías. Como se dijo anteriormente, esto dificulta su comparación y el poder extraer conclusiones desde la síntesis de resultados. Para solventar esto, se revisó con mayor extensión y detenimiento los estudios publicados por los grupos de Gonçalves y Ribeiro, respectivamente, debido a que siguen metodologías y diseños coherentes en todas sus publicaciones. Aún así, es posible establecer ciertas comparaciones entre estudios similares. Por ejemplo, el contenido y la semántica de los logros extraordinarios identificados y codificados por Draucker et al. (2016) en mujeres jóvenes que habían sufrido violencia de pareja, son muy similares a los identificados por Gonçalves, Ribeiro, Mendes, Matos y Santos (2011) en su estudio original a través del cual surge el SCMI.

Los sistemas de codificación del grupo de Gonçalves (SCMI, SCA, SCRA), si bien surgen desde la TN, han sido utilizados para analizar sesiones y casos de modelos terapéuticos diversos. Para la presente revisión se han seleccionado sólo aquellos estudios en los que se aplicaron a TN (exclusivamente o en conjunto con otros modelos). Cuando se han analizado otros modelos de terapia con estos sistemas, los resultados encontrados son similares a los obtenidos en TN. Es por esto que estos sistemas de codificación son considerados transteóricos. Además, es posible afirmar que los logros extraordinarios o las innovaciones narrativas no son un fenómeno exclusivo de la TN, sino que ocurrirían en toda psicoterapia. De hecho, 
esto es lo que suele ocurrir al estudiar factores específicos como responsables del cambio psicoterapéutico. Lo que se suele hallar es que el cambio en una variable psicológica específica suele darse en modelos terapéuticos diversos (Castonguay, 2011), incluso en aquellos en los que dicha variable no se plantea como objeto de intervención. Por ejemplo, un cambio en distorsiones cognitivas no sólo ocurre en TCC, sino que también en terapia interpersonal, farmacoterapia y activación conductual (Dimidjian et al., 2006; Imber et al., 1990; Jacobson et al., 1996; Oei y Free, 1995).

Respecto del SCCT es necesario aclarar que su teorización no viene de la $\mathrm{TN}$, sino que del buscar estudiar las contribuciones de las interacciones terapeutaconsultante a los fenómenos estudiados por el Modelo de Asimilación de Experiencias Problemáticas (APES, Stiles et al., 1990), el SCMI y el SCA. De hecho, el concepto de ZTDP desarrollado por el SCCT proviene del APES y no del modelo de andamiaje de White, aun cuando las conceptualizaciones a las que llega son análogas o equivalentes. En este sentido, sería interesante analizar sesiones de TN con el sistema desarrollado por Ramey, Young y Tarulli (2010) junto con el SCCT, de forma de establecer comparaciones para encontrar similitudes y diferencias.

En esta revisión se han privilegiado estudios sobre procesos de TN en psicoterapia individual, dejando de lado aquellos sobre terapia familiar, terapia de parejas y terapia grupal. Sería interesante revisar este tipo de investigaciones debido a que la TN, desde sus bases relacionales, ha buscado trabajar con familias y parejas.

Por último, la presente revisión no está carente de limitaciones. Al ser una revisión narrativa no ha seguido un criterio sistemático para seleccionar los estudios incluidos. La selección de los mismos se ha hecho a través de buscadores como Google Scholar, Scopus, PubMed y la página web del Dulwich Centre (en la que hay una lista de publicaciones sobre TN), lo que podría sesgar los estudios a los que se ha excedido. Tampoco ha habido una metodología para sintetizar los resultados, por lo que lo discutido a lo largo de este artículo tiene que tomarse meramente como algo informativo. Sin embargo, conceptualmente se logra una síntesis de las principales investigaciones y se logra responder a la pregunta planteada.

Finalmente, las contribuciones prácticas e implicaciones para la práctica clínica que se pueden derivar de la presente revisión, por motivos de extensión, no pueden ser expuestas aquí. El lector interesado puede revisar el artículo de Montesano et al. (2017) en el que se exponen diversas estrategias terapéuticas para la elicitación y trabajo con MI en sus distintos niveles, y el de Oliveira et al. (2016) el que desarrolla estrategias para trabajar con la ambivalencia.

\section{Referencias Bibliográficas}

Beaudoin, M. N., Moersch, M. y Evare, B. S. (2016). The Effectiveness of Narrative Therapy With Children's Social and Emotional Skill Development: An Empirical Study of 813 Problem-Solving Stories. Journal of Systemic Therapies, 35(3), 42-59. https://doi.org/10.1521/jsyt.2016.35.3.42 
Besa, D. (1994). Evaluating Narrative Family Therapy Using Single-System Research Designs. Research on Social Work Practice, 4(3), 309-325. https://doi.org/10.1177/104973159400400303

Braga, C., Ribeiro, A. P., Gonçalves, M. M., Oliveira, J. T., Botelho, A., Ferreira, H. y Sousa, I. (2018). Ambivalence resolution in brief psychotherapy for depression. Clinical Psychology \& Psychotherapy, 25(3), 369-377. https://doi.org/10.1002/cpp.2169

Braga, C., Ribeiro, A. P., Sousa, I. y Gonçalves, M. M. (2019). Ambivalence Predicts Symptomatology in Cognitive-Behavioral and Narrative Therapies: An Exploratory Study. Frontiers in Psychology, 10, 1244. https://doi.org/10.3389/fpsyg.2019.01244

Cashin, A., Browne, G., Bradbury, J. y Mulder, A. (2013). The Effectiveness of Narrative Therapy With Young People With Autism. Journal of Child and Adolescent Psychiatric Nursing, 26(1), 32-41. https://doi. org/10.1111/jcap.12020

Castonguay, L. G. (2011). Psychotherapy, psychopathology, research and practice: Pathways of connections and integration. Psychotherapy Research, 21(2), 125-140. https://doi.org/10.1080/10503307.2011.563250

Dimidjian, S., Hollon, S. D., Dobson, K. S., Schmaling, K. B., Kohlenberg, R. J., Addis, M. E., ... y Jacobson, N. S. (2006). Randomized trial of behavioral activation, cognitive therapy, and antidepressant medication in the acute treatment of adults with major depression. Journal of Consulting and Clinical Psychology, 74(4), 658-670. https://doi.org/10.1037/0022-006X.74.4.658

Draucker, C. B., Smith, C., Mazurczyk, J., Thomas, D., Ramirez, P., McNealy, K., .. y y Martsolf, D. S. (2016). Unique Outcomes in the Narratives of Young Adults Who Experienced Dating Violence as Adolescents. Journal of the American Psychiatric Nurses Association, 22(2), 112-121. https://doi.org/10.1177/1078390315621062

Duncan, B. L., Miller, S. D., Wampold, B. E. y Hubble, M. A. (2010). The Heart \& Soul of Change. Delivering what works in therapy. Washington, DC:American Psychological Association. https://doi.org/10.1037/12075-000

Erbes, C. R., Stillman, J. R., Wieling, E., Bera, W. y Leskela, J. (2014). A Pilot Examination of the Use of Narrative Therapy With Individuals Diagnosed With PTSD. Journal of Traumatic Stress, 27(6), 730-733. https://doi.org/10.1002/jts.21966

Fernández-Navarro, P., Ribeiro, A. P., Soylemez, K. K. y Gonçalves, M. M. (2019). Innovative Moments as Developmental Change Levels: A Case Study on Meaning Integration in the Treatment of Depression. Journal of Constructivist Psychology, O(0), 1-17. https://doi.org/10.1080/10720537.2019.1592037

Fernández-Navarro, P., Rosa, C., Sousa, I., Moutinho, V., Antunes, A., Magalhães, C., ... y Gonçalves, M. M. (2018). Reconceptualization innovative moments as a predictor of symptomatology improvement in treatment for depression. Clinical Psychology and Psychotherapy, 25(6), 765-773. https://doi.org/10.1002/cpp.2306

Foucault, M. (1967). Historia de la locura en la época clásica. México, DF: Fondo de Cultura Económica.

Foucault, M.(1976). Vigilary Castigar. Nacimiento de la presión. Buenos Aires, Argentina: Siglo VeintiunoEditores.

Gelo, O., Pritz, A. y Rieken, B. (2015). Psychotherapy Research: Foundations, Process, and Outcome. Londres, Reino Unido: Springer. https://doi.org/10.1007/978-3-7091-1382-0

Gergen, K. J. y Warhus, L. (2003). La terapia como construcción social. Dimensiones, deliberaciones y divergencias. Revista Venezolana de Psicología Clínica Comunitaria, 3, 13-45.

Gonçalves, M. M., Matos, M. y Santos, A. (2009). Narrative Therapy and the Nature Of "Innovative Moments” in the Construction of Change. Journal of Constructivist Psychology, 22(1), 1-23. https://doi. org/10.1080/10720530802500748

Gonçalves, M. M., Ribeiro, A. P., Mendes, I., Alves, D., Silva, J., Rosa, C., .. y Oliveira, J. T. (2017). Three narrative-based coding systems: Innovative moments, ambivalence and ambivalence resolution. Psychotherapy Research, 27(3), 270-282. https://doi.org/10.1080/10503307.2016.1247216

Gonçalves, M. M., Ribeiro, A. P., Silva, J. R., Mendes, I. y Sousa, I. (2015). Narrative innovations predict symptom improvement: Studying innovative moments in narrative therapy of depression. Psychotherapy Research, 3307(JANUARY), 1-11. https://doi.org/10.1080/10503307.2015.1035355

Gonçalves, M. M., Ribeiro, A. P., Stiles, W. B., Conde, T., Matos, M., Martins, C. y Santos, A. (2011). The role of mutual in-feeding in maintaining problematic self-narratives: Exploring one path to therapeutic failure. Psychotherapy Research, 21(1), 27-40. https://doi.org/10.1080/10503307.2010.507789

Goncalves, M., Ribeiro, A., Mendes, I., Matos, M. y Santos, A. (2011). Tracking novelties in psychotherapy process research: The innovative moments coding system. Psychotherapy Research, 21(5), 1-13. https:// doi.org/10.1080/10503307.2011.560207

Imber, S. D., Pilkonis, P. A., Sotsky, S. M., Elkin, I., Watkins, J. T., Collins, J. F., ... y Glass, D. R. (1990). Modespecific effects among three treatments for depression. Journal of Consulting and Clinical Psychology, 58(3), 352-359. https://doi.org/10.1037/0022-006X.58.3.352 
Jacobson, N. S., Dobson, K. S., Truax, P. A., Addis, M. E., Koerner, K., Gollan, J. K., ... y Prince, S. E. (1996). A component analysis of cognitive-behavioral treatment for depression. Journal of Consulting and Clinical Psychology, 64(2), 295-304. https://doi.org/10.1037/0022-006X.64.2.295

Looyeh, M. Y., Kamali, K. y Shafieian, R. (2012). An Exploratory Study of the Effectiveness of Group Narrative Therapy on the School Behavior of Girls With Attention-Deficit/Hyperactivity Symptoms. Archives of Psychiatric Nursing, 26(5), 404-410. https://doi.org/10.1016/j.apnu.2012.01.001

Lopes, R. T., Gonçalves, M. M., Fassnacht, D. B., Machado, P. P. P. y Sousa, I. (2014). Long-term effects of psychotherapy on moderate depression: A comparative study of narrative therapy and cognitive-behavioral therapy. Journal of Affective Disorders, 167, 64-73. https://doi.org/10.1016/J.JAD.2014.05.042

Lopes, R. T., Gonçalves, M. M., Machado, P. P. P., Sinai, D., Bento, T. y Salgado, J. (2014). Narrative Therapy vs. Cognitive-Behavioral Therapy for moderate depression: Empirical evidence from a controlled clinical trial. Psychotherapy Research, 24(6), 662-674. https://doi.org/10.1080/10503307.2013.874052

Lyotard, J. F. (1994). La condición postmoderna: Informe sobre el saber. Madrid, España: Cátedra.

Madigan, S. (2019). Narrative Therapy (2nd ed.). Washington, DC: American Psychological Association (APA). https://doi.org/10.1037/0000131-000

Matos, M. y Gonçalves, M. (2004). Narratives on marital violence: The construction of change thorugh re-authoring. En R. Abrunhosa, R. Roesch, C. Machado, C. Soeiro y F. Winkel (Eds.), Assessment, intervention and legal issues with offenders and victims (pp. 137-154). Bruselas, Bélgica: Politea.

McAleavey, A. A. y Castonguay, L. G. (2015). The Process of Change in Psychotherapy: Common and Unique Factors. En O. Gelo, A. Pritz y B. Rieken (Eds.), Psychotherapy Research. Foundations, Process, and Outcome (pp. 293-310).Viena, Austria: Springer. https://doi.org/10.1007/978-3-7091-1382-0

McGuinty, E., Armstrong, D., Nelson, J. y Sheeler, S. (2012). Externalizing Metaphors: Anxiety and HighFunctioning Autism. Journal of Child and Adolescent Psychiatric Nursing, 25(1), 9-16. https://doi. org/10.1111/j.1744-6171.2011.00305.x

McKian, S. S., Rabiei, S., Bemana, H. y Ramezani, M. (2019). Experimental design and statistical evaluation on the effect of narrative therapy on body image and body mass index in Iranian overweight and obese women. Obesity Medicine, 14, 100097. https://doi.org/10.1016/j.obmed.2019.100097

Moggia, D. (en prensa). A través del espejo y lo que Tom Andersen encontró allí: Sus contribuciones a la Terapia Familiar y a la Terapia Narrativa. En A. García, L. Rodríguez y H. Maffei Cruz (Eds.), Palabras, Movimientos y Emociones. Nuestro homenaje a Tom Andersen. Chagrin Falls, OH: Taos Institute Publications.

Moggia, D. (2013). ¿Es Efetiva la Terapia Narrativa? En III Encuentro de la Asociación Española de Terapia Narrativa (AETEN). Valencia, España: Universidad de Valencia.

Montesano, A., Oliveira, J. T. y Gonçalves, M. M. (2017). How Do Self-Narratives Change During Psychotherapy? A Review of Innovative Moments Research. Journal of Systemic Therapies, 36(3), 81-96. https://doi. org/10.1521/jsyt.2017.36.3.81

Oei, T. P. S. y Free, M. (1995). Do cognitive behaviour therapies validate cognitive models of mood disorders? A review of the empirical evidence. International Journal of Psychology, 30, 145-179. https://doi. org/10.1080/00207599508246564

Oliveira, J. T., Gonçalves, M. M., Braga, C. y Ribeiro, A. P. (2016). Cómo Lidiar con la Ambivalencia en Psicoterapia: Un Modelo Conceptual para la Formulación de Caso. Revista de Psicoterapia, 27(104), 83-100. https://doi.org/10.33898/rdp.v27i104.122

Payne, M. (2002). Terapia narrativa: una introducción para profesionales. Barcelona, España: Paidós.

Pinto, D., Sousa, I., Pinheiro, A., Freitas, A. C. y Ribeiro, E. (2018). The Therapeutic Collaboration in Dropout Cases of Narrative Therapy: An Exploratory Study. Revista de Psicoterapia, 29(110), 167-184. https:// doi.org/10.33898/rdp.v29i110.209

Poole, J., Gardner, P., Flower, M. C. y Cooper, C. (2009). Narrative Therapy, Older Adults, and Group Work?: Practice, Research, and Recommendations. Social Work With Groups, 32(4), 288-302. https://doi. org/10.1080/01609510902895086

Ramey, H. L., Young, K. y Tarulli, D. (2010). Scaffolding and Concept Formation in Narrative Therapy:AQualitative Research Report. Journal of Systemic Therapies, 29(4), 74-91. https://doi.org/10.1521/jsyt.2010.29.4.74

Ribeiro, A. P., Braga, C., Stiles, W. B., Teixeira, P., Gonçalves, M. M. y Ribeiro, E. (2016). Therapist interventions and client ambivalence in two cases of narrative therapy for depression. Psychotherapy Research, 26(6), 681-693. https://doi.org/10.1080/10503307.2016.1197439

Ribeiro, A. P. y Gonçalves, M. M. (2011). Maintenance and Transformation of Problematic Self-Narratives: A Semiotic-Dialogical Approach. Integrative Psychological and Behavioral Science, 45(3), 281-303. https:// doi.org/10.1007/s12124-010-9149-0 
Ribeiro, A. P., Gonçalves, M. M., Silva, J. R., Brás, A. y Sousa, I. (2015). Ambivalence in Narrative Therapy: A Comparison Between Recovered and Unchanged Cases. Clinical Psychology \& Psychotherapy, 23(2), 166-175. https://doi.org/10.1002/cpp.1945

Ribeiro, A. P, Ribeiro, E., Loura, J., Gonçalves, M. M., Stiles, W. B., Horvath, A. O. y Sousa, I. (2014). Therapeutic collaboration and resistance: describing the nature and quality of the therapeutic relationship within ambivalence events using the Therapeutic Collaboration Coding System. Psychotherapy Research, 24(3), 346-359. https://doi.org/10.1080/10503307.2013.856042

Ribeiro, E., Ribeiro, A. P., Gonçalves, M. M., Horvath, A. O. y Stiles, W. B. (2013). How collaboration in therapy becomes therapeutic: The therapeutic collaboration coding system. Psychology and Psychotherapy: Theory, Research and Practice, 86(3), 294-314. https://doi.org/10.1111/j.2044-8341.2012.02066.x

Silver, E., Williams, A., Worthington, F. y Phillips, N. (1998). Family therapy and soiling: an audit of externalizing and other approaches. Journal of Family Therapy, 20(4), 413-422. https://doi.org/10.1111/1467-6427.00096

Stiles, W. B., Elliott, R., Llewelyn, S. P., Firth-Cozens, J. A., Margison, F. R., Shapiro, D. A. y Hardy, G. (1990). Assimilation of problematic experiences by clients in psychotherapy. Psychotherapy: Theory, Research, Practice, Training, 27(3), 411-420. https://doi.org/10.1037/0033-3204.27.3.411

Vattimo, G. (1986). El fín de la modernidad: Nihilismo y hermenéutica en la cultura posmoderna. Hombre y sociedad. Barcelona, España: Gedisa.

Vromans, L. P. y Schweitzer, R. D. (2011). Narrative therapy for adults with major depressive disorder: Improved symptom and interpersonal outcomes. Psychotherapy Research, 21(1), 4-15. https://doi. org/10.1080/10503301003591792

Vygotsky, L. S. (1980). Mind in Society: The development of higher psychological processes. Boston, MA: Harvard University Press.

Wampold, B. E. (2015). How important are the common factors in psychotherapy? An update. World Psychiatry, 14(3), 270-277. https://doi.org/10.1002/wps.20238

Weber, M., Davis, K. y McPhie, L. (2006). Narrative Therapy, Eating Disorders and Groups: Enhancing Outcomes in Rural NSW. Australian Social Work, 59(4), 391-405. https://doi.org/10.1080/03124070600985970

White, M. (2002). El enfoque narrativo en la experiencia de los terapeutas. Barcelona, España: Gedisa.

White, M. (2016). Mapas de la Práctica Narrativa. Santiago de Chile, Chile: Pranas Chile Ediciones.

White, M. y Epston, D. (1993). Medios narrativos para fines terapéuticos. Barcelona, España: Paidós.

Yoosefi Looyeh, M., Kamali, K., Ghasemi, A. y Tonawanik, P. (2014). Treating social phobia in children through group narrative therapy. The Arts in Psychotherapy, 41(1), 16-20. https://doi.org/10.1016/j.aip.2013.11.005 